\title{
Modelling the range expansion of the Tiger mosquito in a Mediterranean Island accounting for imperfect detection
}

\author{
Giacomo Tavecchia ${ }^{1 *} \mathbb{D}$, Miguel-Angel Miranda ${ }^{2}$, David Borrás ${ }^{2}$, Mikel Bengoa $^{3}$, Carlos Barceló2 \\ Claudia Paredes-Esquivel ${ }^{2}$ and Carl Schwarz ${ }^{4}$
}

\begin{abstract}
Backgrounds: Aedes albopictus (Diptera; Culicidae) is a highly invasive mosquito species and a competent vector of several arboviral diseases that have spread rapidly throughout the world. Prevalence and patterns of dispersal of the mosquito are of central importance for an effective control of the species. We used site-occupancy models accounting for false negative detections to estimate the prevalence, the turnover, the movement pattern and the growth rate in the number of sites occupied by the mosquito in 17 localities throughout Mallorca Island.

Results: Site-occupancy probability increased from 0.35 in the 2012, year of first reported observation of the species, to 0.89 in 2015. Despite a steady increase in mosquito presence, the extinction probability was generally high indicating a high turnover in the occupied sites. We considered two site-dependent covariates, namely the distance from the point of first observation and the estimated yearly occupancy rate in the neighborhood, as predicted by diffusion models. Results suggested that mosquito distribution during the first year was consistent with what predicted by simple diffusion models, but was not consistent with the diffusion model in subsequent years when it was similar to those expected from leapfrog dispersal events.

Conclusions: Assuming a single initial colonization event, the spread of Ae. albopictus in Mallorca followed two distinct phases, an early one consistent with diffusion movements and a second consistent with long distance, 'leapfrog', movements. The colonization of the island was fast, with $~ 90 \%$ of the sites estimated to be occupied 3 years after the colonization. The fast spread was likely to have occurred through vectors related to human mobility such as cars or other vehicles. Surveillance and management actions near the introduction point would only be effective during the early steps of the colonization.
\end{abstract}

Keywords: Tiger mosquito, Site-occupancy model, Population dynamics, Invasion, Range expansion

\section{Background}

Measuring species range expansion and the pattern of dispersal is a central theme in animal ecology and of particular importance in the management or control of invasive species [39]. Most mathematical models for range expansion assume no false negative for detection of a species, that is to say, if a species is present at a given site, it will always be detected [42]. However, cryptic species or species at the initial phase

\footnotetext{
*Correspondence: g.tavecchia@uib.es

'Population Ecology Group, IMEDEA (CSIC-UIB), C. Miquel Marqués 21, 07190 Esporles, Spain

Full list of author information is available at the end of the article
}

of the expansion process, might not be detected under a given density threshold $[6,22]$, which would lead to underestimation of the species prevalence, i.e. number of sites occupied, and the pace of range expansion, i.e. species growth rate. MacKenzie et al. (2006; [26]) proposed an approach based on repeated surveys on sites to estimate the detection probability and the likelihood of species presence accounting for a detection probability $<1$. In contrast to classical models of range expansion [42], site-occupancy models are discrete in space and time. However, their flexibility permits modelling species occurrence as a function of a continuous spatial or temporal 
covariates [16] allowing the comparison of predictions of the pattern of colonization similar to those that characterise classical diffusion models. In a simple diffusion model [10] range dynamic is driven only by the intrinsic population growth rate and by the random short-distance movements of individuals [11, 42]. This model predicts that colonization probability covaries negatively with the distance from the central point or the observed initial site of occupation [27]. In many species, however, random short-distance movements are coupled with long-distance dispersal events, leading to a second type of models characterized by multiple centres of diffusion, an expansion process often referred to as 'hierarchical diffusion' or 'stratified dispersal' $[14,42]$. At a small spatial scale, a negative association between colonization probability and distance from the site of first colonization does not necessarily occur during stratified dispersal because the species can be absent at intermediate distances. However, a diffusion process from each new colonised site would still exist. A third pattern of range expansion is the one resulting from 'leapfrog' dispersal movements, with no or little subsequent diffusion [7]. This model predicts high colonization and extinction probability but low diffusion. In this model, the overall occupancy probability would increase as a result of range expansion but with no apparent relationship with the distance from the initial occupied site and without a clear diffusion process.

We used dynamic site-occupancy models [27] to measure the rate of expansion of the Asian Tiger mosquito Aedes (Stegomya) albopictus (Skuse, 1894) (Diptera; Culicidae) in the Island of Mallorca (Balearic Islands, Spain). We contrasted models consistent with different types of range expansion patterns to investigate the underlying dispersal process. The Asian Tiger mosquito is a daytime-active mosquito native to the tropical and subtropical region of southern Asia [13], and is considered to be one of the most invasive species in the world [23]. Its current distribution includes all continents except Antarctica [20]. In Europe, the species was first detected in Albania in 1979 [1], with no records reported in the rest of the continent until 1990s, when it appeared in Italy [19] from where it rapidly spreads to Southern and Central Europe $[29,41]$. The first detection in mainland Spain occurred in 2004 in Sant Cugat del Vallés (Catalonia, Spain; [2]). Currently its distribution in Spain includes most of the Mediterranean coast as well Northern areas of the Iberian Peninsula ([8], 2016). In Mallorca (Balearic Islands) the species was first detected in 2012 in 5 municipalities [30] and it rapidly spread to Ibiza in 2014 (Barceló et al., 2015) and Menorca in 2016 [3]. Despite being able to feed upon different hosts depending on their availability [45], Ae. albopictus adults obtain blood preferably from humans [32]. The expansion of the Tiger mosquito in Europe has recently created public concerns for its possible role in the transmission of the Zika virus, responsible of microcephaly in newborns of infected mothers (ECDPC, 2016) and as a potential vector for Dengue and Chikungunya viruses [34, 46]. Understanding the spread of invasive mosquito species would thus provide important information needed for a successful control and prevention campaigns. At a large spatial scale, the presence of Ae. Albopictus is associated with the level of rainfall and day time surface temperature and its dispersal is facilitated by human activities [44]. However, pattern of dispersal and distribution at small spatial scale and from the early steps of the colonization process are largely unknown. The isolated character of recently colonized Balearic Islands offers the unique opportunity to follow the invasion process and to determine the mechanisms underlying species diffusion.

Our first aim was to estimate the prevalence of the occupation, i.e. the proportion of sites occupied, and the annual rate of spread, i.e. the proportional change in the number of sites occupied per year. We subsequently investigated the expansion processes by modelling the colonization probability as a linear function of the distance from the site of first reported occupancy. If the expansion followed a diffusion process, we expected the probability of occupancy to abate with the distance from the first reported occupied site. Indeed, diffusion is a slow process for Ae albopictus [28] even when compared with other Aedes species [12]. Also, under random short-distance dispersal, models in which the probability of occupancy at a given site is a function of the occupancy of the neighbourhood would provide a good description of the data [4, 47]. Alternatively, if range expansion occurred mainly by leapfrog dispersal, we expected neither the distance from the initial points nor models depending on neighbourhood covariates to be adequate.

\section{Methods}

Mosquito prevalence and occupancy rate

Mallorca Island is the largest and most populated island of the Balearic archipelago, Eastern Spain, with a surface of $3640 \mathrm{~km}^{2}$ and about $860 \times 10^{3}$ inhabitants (in 2015). Since the first record of Ae. albopictus in Mallorca in 2012, a network of 784 oviposition traps (described in [30]) was deployed in 40 municipalities to monitor the species distribution and range expansion. The monitoring scheme changed over the years resulting in data sparseness. As a consequence, we first restricted the 
analysis to data collected during the 3 months of maximum abundance of Ae. Albopictus (September-November) during the period 2012-2015. To further reduce data sparseness, we used a cluster-by-distance analysis to group neighboring traps into 70 clusters ('sites', hereafter; Fig. 1). The clustering distance threshold was arbitrarily chosen as a good compromise between data richness and number of sites monitored. Besides reducing data sparseness, the clustering allowed a more straightforward interpretation of the yearly occupancy rate because the number and the identity of clusters remained roughly constant throughout the study (Table 1). Nevertheless the dataset was unbalanced and information gaps persisted for some locations (e.g. 23 locations have been sampled in only 1 year). For each site in the dataset we recorded the distance from the location of first observation in the municipality of Bunyola, about $15 \mathrm{Km}$ north the main city of Palma. Clustering and distance analyses were conducted using program R v3.3.1 [38].

\section{Modelling site-occupancy accounting for detection probability}

The occupancy dynamic at each site was investigated using dynamic site-occupancy models $[26,18]$ in which the occurrence of mosquitoes at site $i$ on a given time $t$, $\mathrm{z}_{\mathrm{i}, \mathrm{t}}$, is considered as a latent state governed by the occupancy probability, $\psi_{t}$. Changes in the occupancy over time can be described as in metapopulation dynamics by the extinction, $\varepsilon$, and colonization, $\gamma$, probabilities. Hence the initial occupancy state, at time 1, is assumed to be

$$
z_{\mathrm{i}, 1} \sim \operatorname{Bernoulli}\left(\Psi_{1}\right)
$$

whereas in subsequent period is:

$$
\mathrm{z}_{\mathrm{i}, \mathrm{t}} \mid \mathrm{z}_{\mathrm{i}, \mathrm{t}-\mathrm{1}} \sim \operatorname{Bernoulli}\left(\mathrm{z}_{\mathrm{i}, \mathrm{t}-1}\left[1-\varepsilon_{\mathrm{t}-1}\right]+\left[1-\mathrm{z}_{\mathrm{i}, \mathrm{t}-1}\right] \gamma_{\mathrm{i}, \mathrm{t}-1}\right)
$$

The actual observations, $\mathrm{y}_{\mathrm{i}, \mathrm{t}}$, on a site $i$ at time $t$ are treated as conditional on the occurrence probability and the probability, $\mathrm{p}_{\mathrm{t}}$, to detect the species when present as:

$$
\mathrm{y}_{\mathrm{i}, \mathrm{t}} \mid \mathrm{z}_{\mathrm{i}, \mathrm{t}} \sim \operatorname{Bernoulli}\left(\mathrm{z}_{\mathrm{i}, \mathrm{t}} \mathrm{p}_{\mathrm{t}}\right)
$$

By combining the yearly estimates of $\gamma$ and $\varepsilon$ it is possible to calculate several derived quantities such as i) the probability of occupancy in any given year, $\psi_{\mathrm{t}}=\psi_{\mathrm{t}-1}\left(1-\varepsilon_{\mathrm{t}-1}\right)+\left(1-\psi_{\mathrm{t}-1}\right) \gamma_{\mathrm{t}-1}$ and ii) the proportional increase in the probability of occupancy $\lambda_{t}=\psi_{t+1} / \psi_{t}$ [25]. The proportion of sites occupied at equilibrium, $\psi_{\text {eq }}$, that leads to $\psi_{\mathrm{t}}=\psi_{\mathrm{t}+1}$, can be calculated using the average colonization and extinction probabilities as $\psi_{\text {eq }}=\gamma /(\gamma+\varepsilon)$. In an increasing population typically $\psi_{1}<\psi_{\text {eq }}$ while $\psi_{1}>\psi_{\text {eq }}$ when population is decreasing $[16,37]$.

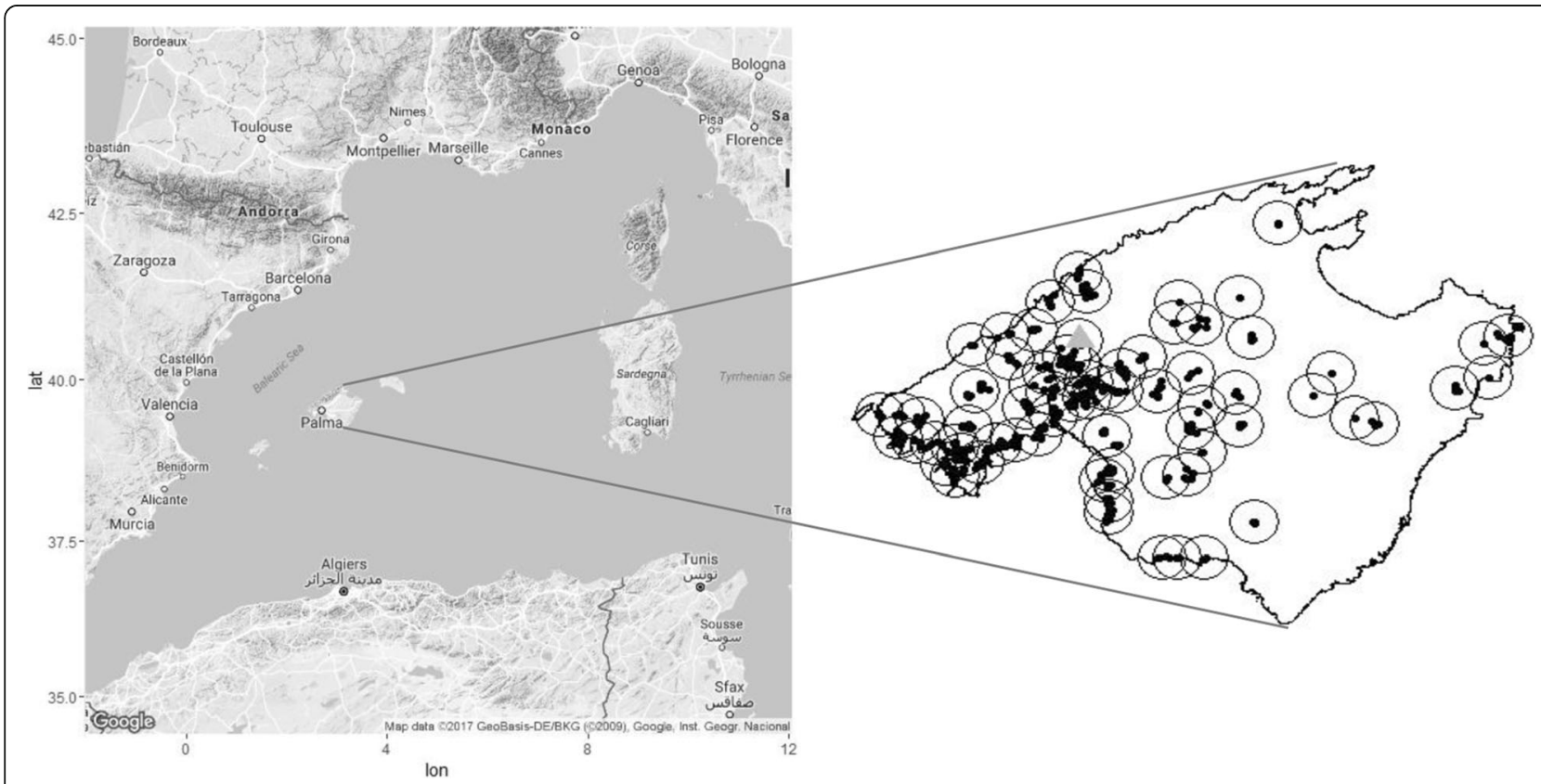

Fig. 1 The Island of Mallorca with the location of the 70 sites (black dots) monitored for the presence of Ae. Albopictus. The circles indicate an area of diameter equal to the average nearest neighbor distance between the sites $(3.6 \mathrm{Km})$. The grey triangle is the site of first observation in 2012. Note that the more eastern locations have been monitored in 2012 only when the species was first reported in Mallorca 
Table 1 Presence-absence data of tiger mosquitoes from 70 unique sites monitored during autumn 2012 to 2015

\begin{tabular}{llll}
\hline Year & Number of unique sites monitored & Observed occupancy rate (\%) & Maximum distance from first reported observation (km) \\
\hline 2012 & 38 & 26 & 21.3 \\
2013 & 39 & 26 & 30.1 \\
2014 & 44 & 57 & 31.6 \\
2015 & 44 & 93 & 41.5 \\
\hline
\end{tabular}

\section{Modelling the pattern of expansion through site-occupancy models}

The presence of $A$. albopictus at each site was first modelled by assuming the initial occupancy, $\psi_{1}$, the extinction, $\varepsilon$, colonization, $\gamma$, and detection, $p$, probabilities varied over time (years), denoted by the model $\psi(\mathrm{t}) \gamma(\mathrm{t}) \varepsilon(\mathrm{t}) \mathrm{p}(\mathrm{t})$. This general model was used to estimate the occupancy rate, the turnover, the occupancy growth rate and the extinction probability over time. The model $\psi(\mathrm{t}) \gamma(\mathrm{t}) \varepsilon(\mathrm{t}) \mathrm{p}(\mathrm{t})$ does not assume any diffusion process and it is consistent with a leapfrog dispersal pattern in which colonization and extinction change over time but without a particular spatial pattern. We then considered a set of models assuming that the observed mosquito range resulted from a diffusion process. We first modelled the initial occurrence, $\psi_{1}$, and the colonization, $\gamma$, probabilities as:

$$
\operatorname{logit}\left(\theta_{i, t}\right)=\alpha_{t}+\beta_{t} X_{i, t}
$$

where $\theta$ refers to the probabilities $\psi_{1}$ or $\gamma$ and $X$ to the distance (standardized) from the site of fist observation (noted 'dist' in model notation). This approach was used by MacKenzie et al. (2006) to model the expansion of the House finch Carpodacus mexicanus (Müller) in North America. Although the model would be consistent with a diffusion process, it does not include the mechanism itself [47]. Following [47], we constrained the colonization probability, $\gamma$, at a given site, $i$, to be dependent on the yearly occupancy rate, $\bar{\psi}_{t}$, within the site neighbourhood, as $\operatorname{logit}\left(\gamma_{i, t}\right)=\alpha_{t}+\beta_{t} \bar{\psi}_{t}$. The autocovariate $\psi_{t}$ is:

$$
\bar{\psi}_{t}^{n}=\frac{1}{l} \sum_{j \varepsilon\left\{n_{i}\right\}} \psi_{j, t}
$$

and it is defined as the probability of occupancy of the neighbouring patch $j$, and $l$ is the number of patches located in the neighbourhood. The neighbourhood can be made by the adjacent patches [4] or by the total patches in the study area as an average measure of the overall occupancy rate [47]. Although at different scales, both models are consistent with either a gradual range expansion through diffusion or a stratified diffusion processes. In theory, if all adjacent cells contribute equally, the two models would only differ in the definition of the neighborhood. In our case, however, this similarity does not hold because the sites monitored were unequally spaced and a further clustering was necessary to define the adjacent sites. To do this, we considered a grid made of $4 \times 4$ $\mathrm{km}$ cells $(n=263)$, a rounded measure of the average nearest neighbour distance between sites. In the majority of the 70 sites monitored $(n=56)$, there was a single site per cell, while 7 cells contained two sites. In this respect the two autoregressive functions cannot be compared because they refer to a different number of sites.

We used a Bayesian framework to estimate model parameters [17]. Bayesian analyses were conducted in WinBUGS [24] using uninformative priors for model parameters (uniform distribution from -20 to +20 for linear predictors and 0 to 1 for probabilities). The posterior distributions of parameters were sampled, using 3 chains and 25,000 simulations (the first 5000 discarded as a burnin period). Model selection in Bayesian analyses is not straightforward [43]. Across nested models, selection can be done using the Deviance Information Criterion (DIC), a generalisation of the Akaike's Information Criterion (AIC; [5]) for hierarchical models. However, comparisons between models with and without an autoregressive structure cannot be performed using the DIC because the DIC is computed at different levels in the hierarchy of data and parameters. Model adequacy was thus assessed by inspecting the estimates and their standard deviations. We report the DIC of all models, but we warn readers that this should not be taken as a strict criterion for model explanatory power.

\section{Results}

\section{Mosquito prevalence and occupancy rate}

The observed edge of the mosquito distribution, i.e. the site at the greatest distance from the initial reported occupancy, was expanding during the study period at an average rate of $6.1 \mathrm{~km}$ per year (Fig. 2). Observed site occupancy rate assuming a detection probability of 1.00 were $0.26 \quad(n=38), 0.26 \quad(n=39), 0.57 \quad(n=44)$ and $0.93(n=44)$ in 2012, 2013, 2014 and 2015, respectively (Table 1). However, the site-occupancy model, $\psi(\mathrm{t}) \gamma(\mathrm{t}) \varepsilon(\mathrm{t}) \mathrm{p}(\mathrm{t})$, assuming all parameters time-dependent 


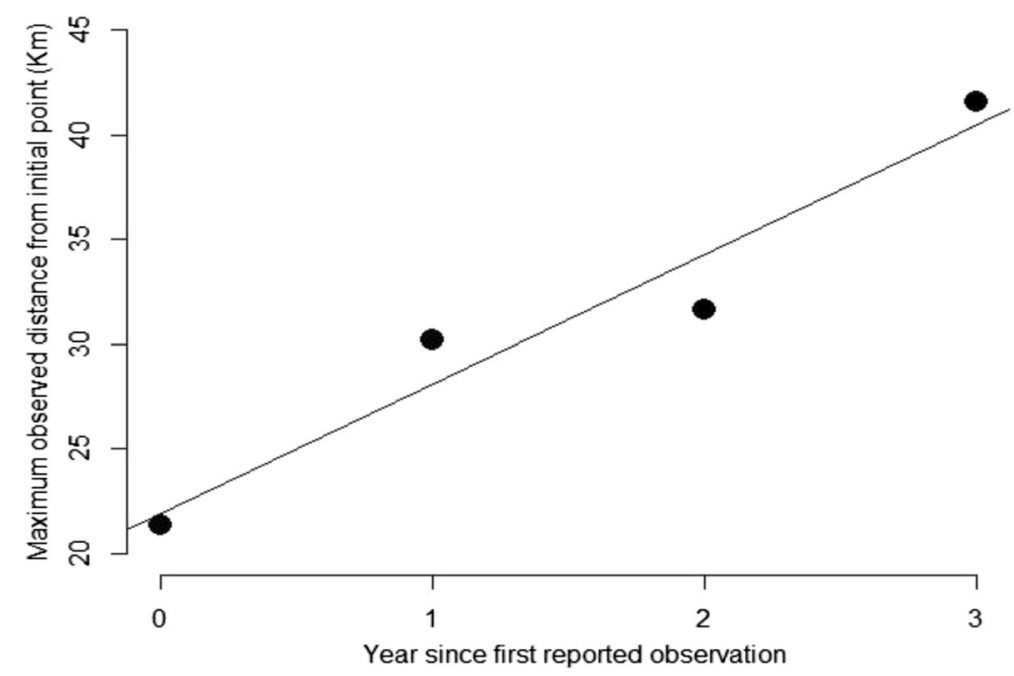

Fig. 2 Maximum distance of a observed occurrence from the point of first observation by year. The solid line indicates the expected values assuming a linear diffusion. The speed of the observed expansion is $6.2 \mathrm{~km}$ per year (slope of the regression line)

revealed that the detection probability varied from 0.23 in 2013 to 0.85 in 2015 , being less than 1 in all years (Table 2). As expected, this model led to estimated occupancy probabilities higher than those observed $(0.35,0.59,0.57$ and 0.90 in 2012, 2013, 2014 and 2015, respectively; Table 2). The probability of local extinction was generally high (average 0.38), but it dropped to 0.07 in 2015 (Table 2). The initial occupancy rate in $2012\left(\psi_{2012}=0.351\right)$ was lower than the expected occupancy rate at equilibrium $\left(\psi_{\text {eq }}=0.661\right)$ confirming the observed range expansion over the study area. The average growth rate in the occupancy probability was 1.50 , equivalent to a $50 \%$ increase per year in the number of sites occupied by the species per year. However, this rate of increase was not constant and the range expansion greatly increased after the initial colonization and during the last year (periods 2012-2013 and 2014-2015, Table 2).

\section{Modelling the pattern of expansion through site-occupancy model}

The DIC of the general model (DIC model 2 $\psi(t) \gamma(t) \varepsilon(t) p(t)=353.89)$ improved slightly when the initial probability of occupancy was model as a function of the distance from the point of first reported observation (DIC model $1 \psi($ dist $) \gamma(\mathrm{t}) \varepsilon(\mathrm{t}) \mathrm{p}(\mathrm{t})=352.29$; Tab. 3). The estimates of $\alpha$ and $\beta(\alpha=-3.581, \beta=-5.537)$ with the upper $95 \%$ credible intervals of $\beta$ lower than 0.00 $(+95 \% \mathrm{CI}=-1.978)$ indicate that the probability of occupancy in the first year declined as a function of distance. Estimates showed a sharp decline in the occupancy rate, with no occupancy expected at mid-distance between the first reported observation and the farthest site monitored (c. $23 \mathrm{~km}$; Fig. 3). However, the probability of colonization of empty patches in subsequent years did not depend on the distance from the first reported observation (DIC $\psi($ dist $) \gamma($ dist $) \varepsilon(\mathrm{t}) \mathrm{p}(\mathrm{t})=362.03$; Table 3-

Table 2 Modelling the occupancy dynamics of the tiger mosquito in Mallorca Island. $\psi=$ occupancy probability, $\gamma=$ colonization probability, $\varepsilon=$ extinction probability. Effects: $\mathrm{t}=$ time effect, dist $=$ distance from the site of first observation, $\mathrm{m} . \psi=$ autocovariate based on the average occupancy rate in the whole area, $\mathrm{D}=$ autocovariate based on the adjacent occupancy rate (see details in 'Methods'). Note that $\psi$ (covariate) refers to occupancy rate in 2012 only, the occupancy probabilities for the subsequent years are calculated as derived parameters (see text for details)

\begin{tabular}{|c|c|c|c|c|c|}
\hline Model & Type of dispersal & Autocovariate & Notation & $\mathrm{DIC}$ & Reference \\
\hline 1 & Initial diffusion + leapfrog & No & $\psi(\operatorname{dist}) \gamma(\mathrm{t}) \varepsilon(\mathrm{t}) \mathrm{p}(\mathrm{t})$ & 352.29 & [26] \\
\hline 2 & Leapfrog & No & $\psi(t) \gamma(t) \varepsilon(t) p(t)$ & 353.89 & [26] \\
\hline 3 & Diffusion & No & $\psi($ dist $) \gamma($ dist $) \varepsilon(t) p(t)$ & 362.03 & [26] \\
\hline 4 & Diffusion & No & $\psi(\mathrm{t}) \gamma(\operatorname{dist}) \varepsilon(\mathrm{t}) \mathrm{p}(\mathrm{t})$ & 363.84 & [26] \\
\hline 5 & Leapfrog / Diffusion & Yes & $\psi(\mathrm{t}) \gamma(\mathrm{m} . \psi) \varepsilon(\mathrm{t}) \mathrm{p}(\mathrm{t})$ & 374.32 & [47] \\
\hline 6 & Stratified Diffusion & Yes & $\psi(\mathrm{t}) \gamma(\mathrm{D}) \varepsilon(\mathrm{t}) \mathrm{p}(\mathrm{t})$ & 406.18 & [4] \\
\hline
\end{tabular}




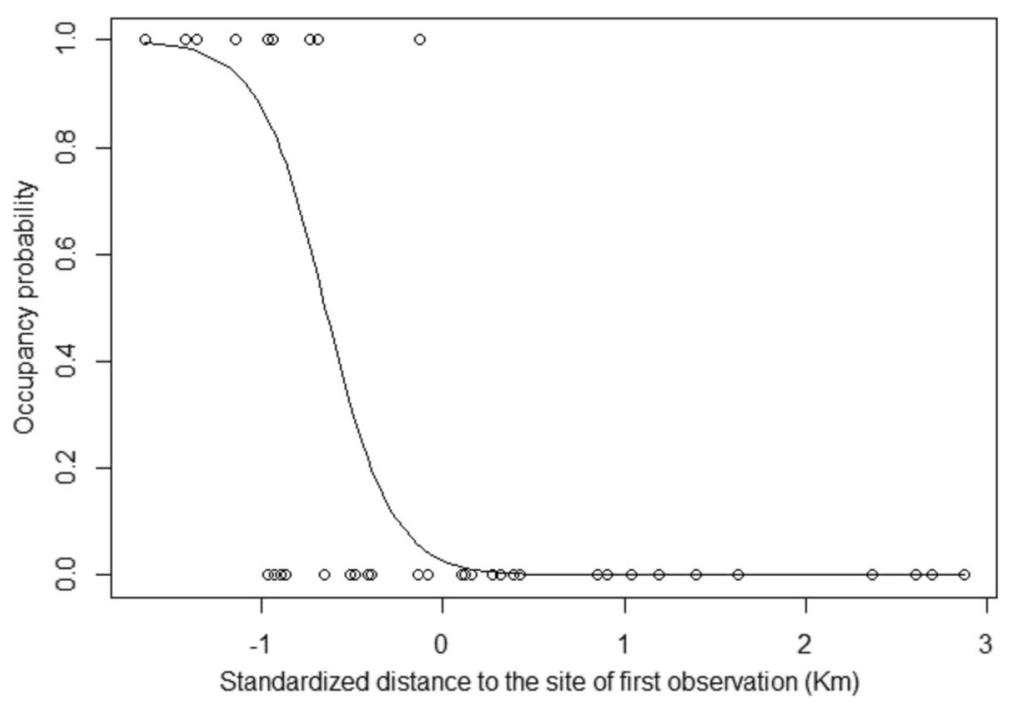

Fig. 3 Predicted (solid line) and observed (circle) site occupancy probability in relation to the distance from the first reported observation (estimates from model assuming an initial diffusion followed by leapfrog movements, noted $\psi($ dist $) \gamma(t) \varepsilon(t) p(t)$ )

Table 3 Estimates from model $\psi(t) \gamma(t) \varepsilon(t) p(t)$, assuming all parameters variable over time. Credible interval $(\mathrm{Cl})$ at $2.5 \%$ and 97.5\% are reported. Parameters: $\psi_{i}=$ occupancy probability at time $i, \gamma_{i}=$ colonization probability, e.g. the probability that an empty site is occupied between $i$ and $i+1, \varepsilon_{\mathrm{i}}=$ extinction probability, e.g. the probability that an occupied size at $i$ is not-occupied at $i+1_{1,} \lambda_{\mathrm{i}}=$ the growth rate of occupied size between $i$ and $i+1, \psi_{\text {eq }}=$ occupation probability at equilibrium (see text for details)

\begin{tabular}{lllll}
\hline Parameter & Mean & $\mathrm{Sd}$ & $2.5 \%$ quantile & $97.5 \%$ quantile \\
\hline$\psi_{2012}$ & 0.351 & 0.102 & 0.184 & 0.58 \\
$\psi_{2013}$ & 0.587 & 0.159 & 0.291 & 0.887 \\
$\psi_{2014}$ & 0.568 & 0.071 & 0.428 & 0.704 \\
$\psi_{2015}$ & 0.899 & 0.044 & 0.800 & 0.968 \\
$\gamma_{2012}$ & 0.667 & 0.201 & 0.258 & 0.980 \\
$\gamma_{2013}$ & 0.669 & 0.182 & 0.251 & 0.974 \\
$\gamma_{2014}$ & 0.862 & 0.075 & 0.686 & 0.973 \\
$\varepsilon_{2012}$ & 0.566 & 0.24 & 0.059 & 0.935 \\
$\varepsilon_{2013}$ & 0.509 & 0.133 & 0.252 & 0.769 \\
$\varepsilon_{2014}$ & 0.073 & 0.051 & 0.007 & 0.197 \\
$p_{2012}$ & 0.63 & 0.118 & 0.384 & 0.839 \\
$p_{2013}$ & 0.26 & 0.103 & 0.112 & 0.510 \\
$p_{2014}$ & 0.80 & 0.048 & 0.693 & 0.882 \\
$p_{2015}$ & 0.85 & 0.033 & 0.782 & 0.909 \\
$\lambda_{2012}$ & 1.846 & 0.833 & 0.685 & 3.867 \\
$\lambda_{2013}$ & 1.057 & 0.388 & 0.580 & 2.047 \\
$\lambda_{2014}$ & 1.610 & 0.220 & 1.261 & 2.113 \\
$\psi_{\text {eq }}$ & 0.661 & 0.066 & 0.548 & 0.804 \\
\hline
\end{tabular}

Fig. 4). Models including an autoregression structure in which colonization probabilities were modelled as a linear function of the average neighbouring occupancy rate in the form $\alpha+\beta_{1 t} \bar{\psi}_{t}^{n}$ delivered positive but unrealistic standard deviations for the $\beta$ s parameters (mean \pm sd: $\beta_{1,2013}=1.91 \pm 11.3, \beta_{1,2014}=4.637 \pm 9.51$ and $\beta_{1,2015}$ $=-6.667 \pm 9.46)$. Similar imprecise estimates were obtained when only adjacent cells were considered (Fig. 5).

\section{Discussion}

\section{The expansion of the tiger mosquito}

The range expansion of the Tiger mosquito in the island of Mallorca has been rapid, with an estimated occupancy of monitored sites probability that increased from 0.35 in 2012 to nearly 0.90 in 2015 and an average annual growth rate in the occupancy of 1.50. Interestingly, local extinction probability was relatively high (except from 2014 to 2015) suggesting a high turnover in the occupied sites. During the first year, when the number of mosquitos was presumably small, treatments with insecticides by private citizens and local administration might have caused temporary extinction of the species in some monitored sites. At present we ignore the intensity and influence of these actions. Extinctions can also have occurred naturally because newly colonized locations are expected to be occupied by a small number of mosquitos. However, colonization probability was also high, leading to a fast re-colonization of locations from which the species disappeared. Under a simple diffusion model [42], the colonization probability should negatively covary with the distance from the site of first observation (MacKenzie et al. 2006). Our results indicated that the 


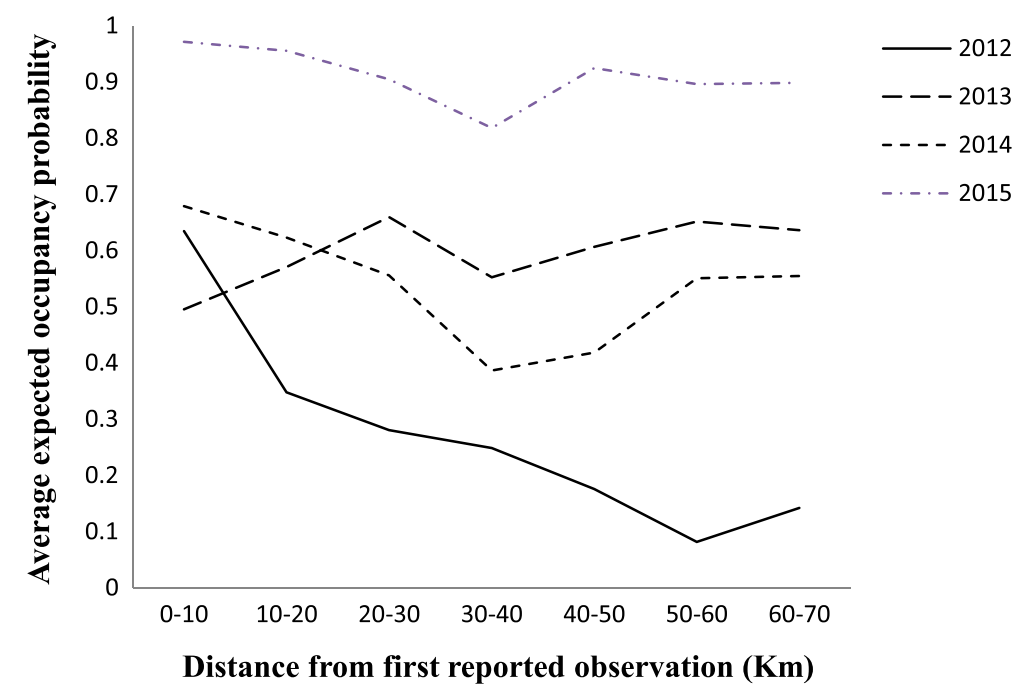

Fig. 4 Average site occupancy probability in relation to the distance from the first reported observation (estimates from the model in which all parameters were time-dependent, noted $\psi(t) \gamma(\mathrm{t}) \varepsilon(\mathrm{t}) \mathrm{p}(\mathrm{t}))$

distribution of occupied sites during the first year responded to what predicted from a simple diffusion model with random short dispersal movements. In contrast, from 2013 to 2015 the colonization of new sites did not occur as a diffusion process, at least not at the spatio-temporal scale considered here. The fast range expansion is better described by leapfrog dispersal movements, in which an increasing number of sites are occupied each year, but without a clear relationship with the distance from the initial colonization. Autoregressive models delivered unrealistic standard deviations of parameter estimates indicating that a gradual diffusion process is unlikely to have shaped the current distribution at least at the spatial scale considered here. Collantes et al. 2015 [8] mentioned a possible diffusion process of dispersal of $A$. albopictus from its first detection site in mainland Spain in 2004. However, no analyses were conducted for demonstrating such type of dispersal. Other authors also reported leapfrog dispersal movements at larger spatial scale $(>500 \mathrm{~km})$ from the site of first detection in Catalonia to the Valencia region (Bueno-Marí et al., 2013). The same pattern of progressive and gradual invasion since the initial point combined with sporadic "jumps" has been also proposed by Roche et al. [35] on a study of the distribution of $A e$. albopictus in continental France and Corsica. In comparison to other mosquitos, Ae. albopictus show a low dispersal capability [12]. According to Marini et al. [28], for example, the average flight distance of Ae. albopictus is $119 \mathrm{~m}$ per day. However, passive transportation of eggs, through translocations of used tires [33] and adults mosquitoes in vehicles [32] are probably the mechanisms of leapfrog dispersal movements. Despite our conclusions are drawn on a smaller spatial scale than the one previously considered, they are in agreement with what is known of the colonization pattern of the species. However, they are based on the assumption of a single initial colonization event in 2012. We cannot exclude that subsequent colonization (i.e. independent introductions; see for example $[15,36]$ ) occurred after 2012. At the moment it is unknown whether or where this happened, but multiple introductions from mainland through the local airport or the two main ports of Alcudia and Palma would be consistent with a stratified diffusion as predicted by models with auto-covariates The change in colonization and extinction probability in the last year of the study might partly be due to natural causes. For example the amount of rainfall during the summer 2015 was particularly high and can partially explain the high colonization and recapture probabilities (see below). The short period of the study does not permit to fully investigate a relationship between colonization and rainfall but an important role of weather variables has been found in the oviposition dynamics of Ae. aegypti in Northwestern Argentina [9] and in the abundance of Ae albopictus in the French Riviera (Tar et al. 2013).

\section{Site occupancy models and species range expansion}

The pattern of range expansion of any given species depends on several characteristic such as landscape heterogeneity [11], interspecific competition ([47], 2014), species life-history traits [22], climatic and humanrelated factors $[36,40]$.

It is not surprising that analytical models of range expansion are a necessary oversimplification of the underlying biological processes. They allow, nevertheless, some generalizations and the estimates of important 


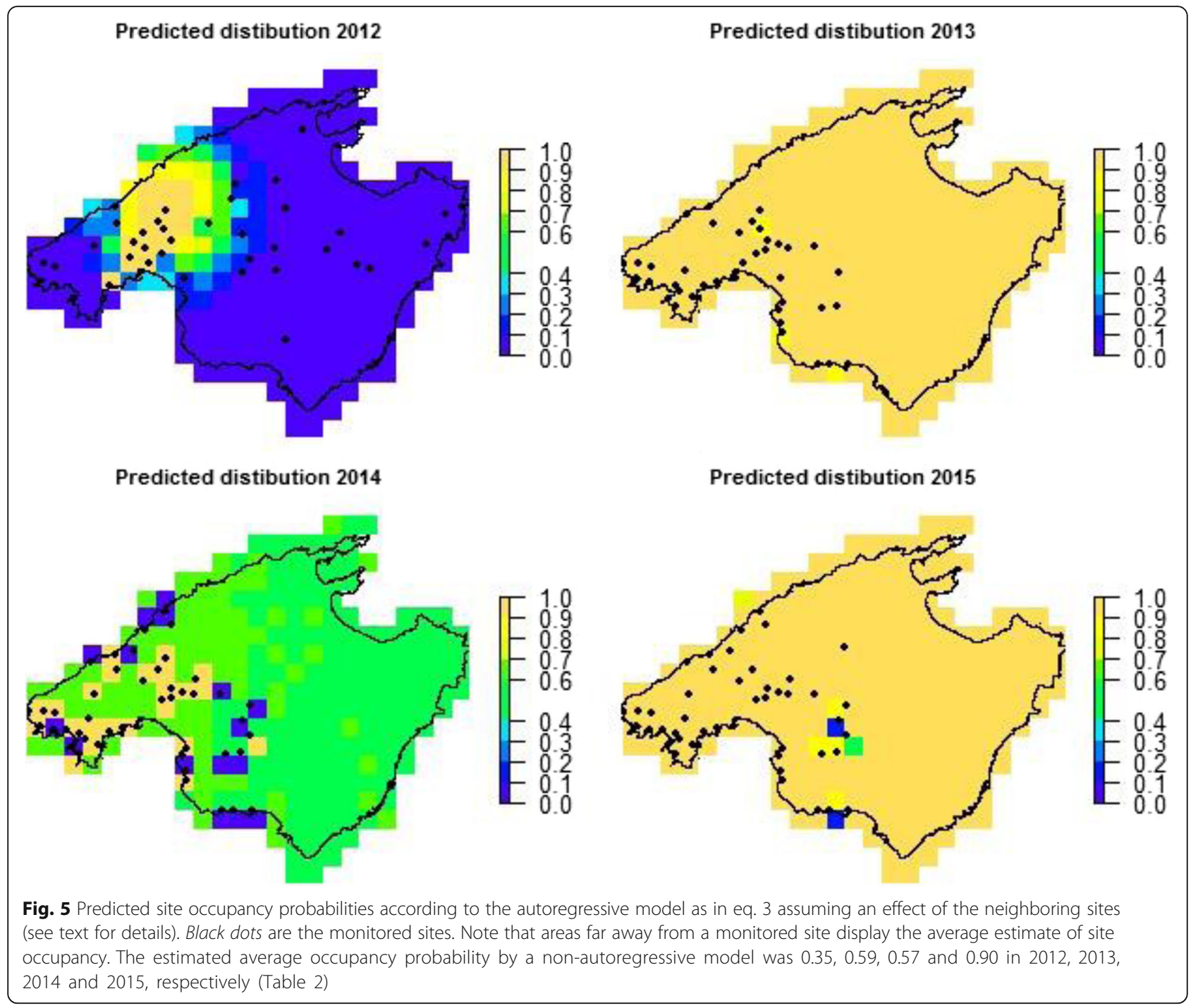

parameters that modulate the range expansion [22]. Here we used dynamic site-occupancy models to estimate occupancy rate and colonization speed of the Tiger mosquito accounting for imperfect detection. In contrast to classical models [14], site-occupancy models are discrete in space and time. However, we constrained parameter variability as a function of a site-dependent covariate to deliver predictions consistent with different patterns of colonization as in classical continuous models, i.e. 'leapfrog' versus 'diffusion'. A clear limitation of our work was the difficulty in finding criteria for model selection. However, the problem of contrasting hierarchical models is not only limited to the present study and it is a topic under study in statistical theory and numerical ecology [43]. Additional problems derived from the fast expansion of the mosquito, the limited number of sites monitored and/or a possible spatially consisted driver of the colonization probability. These factors contribute to reduce the variability in occupancy rate among sites leading to numerical problems in model fitting when estimating the effect of the covariate. Beside these limitations, we showed the potential of site-occupancy models in estimating range expansion parameters [26] and can be very useful in the study of disease prevalence and vector dynamics [21, 31]. For example, Padilla-Torres et al. [31] used site-occupancy models to study the prevalence of Ae. aegypti and Ae. albopictus. They concluded that routine surveillance based on rapid larval surveys led to a lower prevalence of both species and suggest a combined used of ovitrap-based surveillance with analytical methods based on imperfect detection. Finally, MacKenzie and Nichols [27] treated occupancy as a surrogate of abundance. In our case mosquito abundance is more likely to be reflected in the probability of detection, which can be seen as the probability of a trap being used by a gravid female. This is because the conditional probability to detect mosquito larvae in the oviposition traps given 
that a female has used the trap is equal to 1.00. This would explain why in 2015, when the probability of recapture was high (0.85), the extinction probability was low (0.07). However, the link between abundance and detection is not straightforward because it would depend on multiple factors that have not been considered here, i.e. the habitat characteristics or the availability of alternative breeding sites. The present work is more descriptive than predictive and future research should incorporate additional site-dependent covariates in the models such as habitat type and site attractiveness. This can be done with static (opposite to 'dynamic') single-season occupancymodel. Single season models would not allow investigating the expansion process as we did here, but they will avoid trap-clustering and would permit to model mosquito presence using fine scale habitat covariates to predict future distributions.

\section{Conclusions}

Assuming a single colonization event in 2012, we concluded that the rapid expansion of Ae. Albopictus in Mallorca Island occurred in two phases. In a first phase the distribution appeared consistent with a diffusion process. This was rapidly followed by leap-frog dispersal events that resulted in an estimated occupancy probability of $90 \% 3$ years after the colonization. The two distinct phases imply that surveillance and management actions near the introduction point would only be effective during the early steps of the colonization. The lowest extinction probability was recorded in the year with the highest amount of summer rainfall suggesting a role of weather covariates on the paste of the expansion. Dynamic siteoccupancy models offer a robust analytical framework for the study of range expansion. They are particularly suitable for the study of cryptic species with high turnover as they permit to frame imperfect detections.

\section{Abbreviations}

AIC: Akaike's Information Criterion; DIC: Deviance Information Criterion

\section{Acknowledgements}

We are grateful to all volunteers who helped site monitoring from 2012 to 2015. Thanks to M. Kery and F. Bled for their help in modelling the data and to A. Sanz-Aguilar for her comments on an early draft of the manuscript. Many thanks to G. Guillera-Arroita and J. Lahoz-Monfort for an early discussion about the analysis. We thank two anonymous referees for the constructive comments that have helped to improve the clarity and quality of the work. GT is grateful to I. Hendriks for her support. The occupancy data used in this analysis are available at http://cedai.imedea.uib-csic.es/geonetwork/srv/es/main.home under the terms and conditions specified by the CEDAl database platform.

\section{Funding}

GT has been supported by a "Salvador de Madariaga" fellowship (Spanish Minister of Education, Culture and Sport, Ref.: PRX16/00101) for the mobility of researchers.

\section{Authors' contributions}

MAM, DB, MB, CPE collected the data and contributed to discussion. GT and CS prepared the data and performed the statistical analyses. All authors have contributed to the editing of the manuscript, read and approved the final version of the text.

Ethics approval and consent to participate

Monitoring has been conducted in respects of ethical issues.

Competing interests

Authors do not have any competing interests concerning this study.

\section{Consent for publication}

All data have been collected by the authors.

\section{Publisher's Note}

Springer Nature remains neutral with regard to jurisdictional claims in published maps and institutional affiliations.

\section{Author details}

${ }^{1}$ Population Ecology Group, IMEDEA (CSIC-UIB), c. Miquel Marqués 21, 07190 Esporles, Spain. ${ }^{2}$ Laboratory of Zoology, Department of Biology, University of the Balearic Island, c. Valldemossa s/n, Palma de Mallorca, Spain. ${ }^{3}$ Consultoria Moscard Tigre, c. Gremi Passamaners 24, Local 15, 07009 Palma de Mallorca, Spain. ${ }^{4}$ Department of Statistics and Acutarian Science, Simon Fraser University, Burnaby, BC, Canada.

Received: 22 February 2017 Accepted: 15 June 2017

Published online: 27 July 2017

\section{References}

1. Adhami J, Reiter P. Introduction and Establishment of Aedes (Stegomyia) Albopictus Skuse (Diptera: Culicidae) in Albania article. J Am Mosq Control Assoc. 1998:14(3):340-3.

2. Aranda C, Eritja R, Roiz D. First record and Establishment of the mosquito Aedes albopictus in Spain. Med Vet Entomol. 2006;20:150-2. doi:10.1111/j. 1365-2915.2006.00605.x.

3. Bengoa M, Delacour-Estrella S, Barceló C, Paredes-Esquivel C, Leza M, Lucientes J, Molina R, Ángel Miranda M. First Record of Aedes albopictus (Skuse, 1894) (Diptera; Culicidae) from Minorca (Balearic Islands, Spain). J Am Mosq Control Assoc. 2016;34:5-9.

4. Bled F, Andrew Royle J, Cam E. Hierarchical Modeling of an invasive spread: the Eurasian collared-dove Streptopelia decaocto in the United States. Ecol Appl. 2011;21(1):290-302. doi:10.1890/09-1877.1.

5. Burnham KP, Anderson DR. Model selection and inference. A practical information-theoretic approach. 2nd ed. New York: Springer; 2002.

6. Carey JR. The Incipient Mediterranean Fruit Fly Population in California: Implications for Invasion Biology. Ecology. 1996;77(6):1690-97.

7. Clobert J, Danchin E, Nichols JD, Dhondt AA. Dispersal. New York: Oxford University Press; 2001.

8. Collantes F, Delacour S, Alarcón-Elbal PM, Ruiz-Arrondo I, Delgado JA Torrell-Sorio A, et al. Review of ten-years presence of Aedes albopictus in Spain 2004-2014: known distribution and public Health concerns. Parasit Vectors. 2015;8(1):655. doi:10.1186/s13071-015-1262-y.

9. Estallo EL, Ludueña-Almeida FF, Introini MV, Zaidenberg M, Almirón WR Weather variability associated with Aedes (Stegomyia) Aegypti (Dengue vector) Oviposition dynamics in northwestern Argentina. PLoS One. 2015: 10(5):e0127820. doi:10.1371/journal.pone.0127820.

10. Fisher RA. The wave of advance of advantageous genes. Ann Hum Genet. 1937:7:355-69.

11. Fraser EJ, Lambin X, Travis JMJ, Harrington LA, Palmer SCF, Bocedi G, et al. Range expansion of an invasive species through a heterogeneous landscape - the case of American mink in Scotland. Divers Distrib. 2015; 21(8):888-900. doi:10.1111/ddi.12303.

12. Goubert C, Minard G, Vieira C, Boulesteix M. Population genetics of the Asian Tiger mosquito Aedes albopictus, an invasive vector of human diseases. Heredity. 2016;117(3):125-34.

13. Hawley WA. The Biology of Aedes albopictus. J Am Mosq Control Assoc. 1988;1:1-39.

14. Hengeveld R. Dynamics of biological invasions. Springer Science \& Business Media. 1989.

15. Kamgang B, Brengues C, Fontenille D, Njiokou F, Simard F, Paupy C. Genetic structure of the Tiger mosquito, Aedes albopictus, in Cameroon (Central Africa). PLoS One. 2011;6(5):e20257. 
16. Kéry M, Guillera-Arroita G, Lahoz-Monfort JJ. Analysing and mapping species range dynamics using occupancy models. J Biogeogr. 2013;40(8):1463-74. doi:10.1111/jbi.12087.

17. Kery M, Royle JA. Applied Hierarchical Modeling in Ecology: Analysis of Distribution, Abundance and Species Richness in R and BUGS: Volume 1: Prelude and Static Models. London: Academic Press; 2015.

18. Kéry M, Royle JA. Hierarchical Modelling and Estimation of Abundance and Population Trends in Metapopulation Designs'. Journal of Animal Ecology. 2010;79(2):453-61.

19. Knudsen AB, Romi R, Majori G. Occurrence and spread in Italy of Aedes albopictus, with implications for its introduction into other parts of Europe. J Am Mosq Control Assoc. 1996;12(2):177-83.

20. Kraemer MUG, Sinka ME, Duda KA, Adrian Q N Mylne, Freya M. Shearer, Christopher M. Barker, Chester G. Moore, et al. The global distribution of the Arbovirus vectors Aedes aegypti and Ae. Albopictus' elife. 2015; doi:10.7554/elife.08347.

21. Lachish S, Gopalaswamy AM, Knowles SCL, Sheldon BC. Site-occupancy modelling as a novel framework for assessing test sensitivity and estimating wildlife disease prevalence from imperfect diagnostic tests. Methods Ecol Evol. 2012;3:339-48.

22. Lockwood JL, Hoopes MF, Marchetti MP. Invasion Ecology. John Wiley \& Sons, 2009

23. Lowe S, Browne M, Boudjelas S, De Poorter M. 100 of the World's Worst Invasive Alien Species A selection from the Global Invasive Species Database. Published by The Invasive Species Specialist Group (ISSG) a specialist group of the Species Survival Commission (SSC) of the World Conservation Union (IUCN). 2000. 12pp.

24. Lunn DJ, Thomas A, Best N, Spiegelhalter D. WinBUGS - a Bayesian Modelling framework: concepts, structure, and extensibility. Stat Comput. 2000;10:325-37. doi:10.1023/A:1008929526011.

25. MacKenzie DI, Nichols JD. 'Occupancy as a Surrogate for Abundance Estimation'. Animal Biodiversity and Conservation. 2004;27:461-67

26. Mackenzie DI, Nichols J, Royle JA, Pollock KH, Bailey LL, Hines JH. Occupancy Estimation and Modeling: Inferring Patterns and Dynamics of Species Occurrence. New York: Academic Press; 2006

27. MacKenzie DI, Nichols JD, Hines JE, Knutson MG, Franklin AB. Estimating site-occupancy colonization and local extinction when a species is detected imperfectly. Ecology. 2003:84(8):2200-7. doi:10.1890/02-3090.

28. Marini F, Caputo B, Pombi M, Tarsitani G, Della Torre A. Study of Aedes albopictus dispersal in Rome, Italy, using sticky traps in mark-releaserecapture experiments. Med Vet Entomol. 2010;24(4):361-8. doi:10.1111/j. 1365-2915.2010.00898.x.

29. Medlock JM, Hansford KM, Schaffner F, Versteirt V, Hendrickx G, Zeller H, et al. A review of the invasive mosquitoes in Europe: ecology, public Health risks, and control options. Vector Borne Zoonotic Dis. 2012;12(6): 435-47. doi:10.1089/vbz.2011.0814.

30. Miquel M, Río R, Borràs D, Barceló C, Paredes-Esquivel C, Lucientes J. First Detection of Aedes albopictus (Diptera: Culicidae) in the Balearic Islands (Spain) and Assessment of Its Establishment according to the ECDC Guidelines. J Am Mosq Control Assoc. 2013;31:8-11

31. Padilla-Torres, Samael D., Gonçalo Ferraz, Sergio L. B. Luz, Elvira ZamoraPerea, and Fernando Abad-Franch. 2013. 'Modeling Dengue Vector Dynamics under Imperfect Detection: Three Years of Site-Occupancy by Aedes aegypti and Aedes albopictus in Urban Amazonia'. PLOS ONE 8 (3).

32. Paupy C, Delatte H, Bagny L, Corbel V, Fontenille D. Aedes albopictus, an Arbovirus vector: from the darkness to the light. Microbes Infect. 2009; 11(14):1177-85. doi:10.1016/j.micinf.2009.05.005.

33. Reiter $P$, Sprenger D. The used tire trade: a mechanism for the worldwide dispersal of container breeding mosquitoes. J Am Mosq Control Assoc. 1987;3(3):494-501.

34. Rezza G. Aedes albopictus And the reemergence of Dengue. Public Health. 2012;12:72.

35. Roche B, Léger L, L'Ambert G, Lacour G, Foussadier R, BEsnard G, et al. The spread of Aedes albinopictus in metropolitan France: contribution of environmental drivers and human activities and predictions for a near future. PLoS One. 2015;10(5):e0125600.

36. Rochlin I, Ninivaggi DV, Hutchinson ML, Farajollahi A. Climate change and range expansion of the Asian Tiger mosquito (Aedes albopictus) in northeastern USA: implications for public Health practitioners. PLoS One. 2013;8(4):e60874.
37. Royle JA, Nichols JD, Kéry M. Modelling occurrence and abundance of species when detection is imperfect. Oikos. 2005;110(2):353-9. doi:10. 1111/j.0030-1299.2005.13534.x.

38. R Core Team. R: A language and environment for statistical computing. $\mathrm{R}$ Foundation for Statistical Computing, Vienna, Austria. 2016. http://www.Rproject.org/.

39. Sakai AK, Allendorf FW, Holt JS, Lodge DM, Molofsky J, With KA, et al. The population Biology of invasive specie. Annu Rev Ecol Syst. 2001;32:305-32.

40. Semenza JC. Climate Change and Human Health, International Journal of Environmental Research and Public Health. 2014;11:7347-53.

41. Scholte EJ, Schaffner F. 'Waiting for the Tiger: Establishment and Spread of the Aedes albopictus Mosquito in Europe'. In Emerging Pests and Vector-Borne Diseases in Europe. Ecology and Control of Vector-Borne Diseases (1), edited by Taken W. and Knols B. Wageningen Academic publishers. 2007. 241-60.

42. Shigesada N, Kawasaki K. Biological invasions: theory and practice. UK. Oxford University Press; 1997.

43. Tenan S, O'Hara RB, Hendriks I, Tavecchia G. Bayesian model selection: the Steepest Mountain to climb. Ecol Model. 2014;283(July):62-9. doi:10.1016/j. ecolmodel.2014.03.017.

44. Tran A, L'Ambert G, Lacour G, Benoît R, Demarchi M, Cros M, et al. A rainfalland temperature-driven abundance model for Aedes albopictus populations. Int J Environ Res Public Health. 2013;10(5):1698-719. doi:10.3390/ ijerph10051698.

45. Valerio L, Marini F, Bongiorno G, Facchinelli L, Pombi M, Caputo B, Maroli M, della Torre A. Host-feeding patterns of Aedes albopictus (Diptera: Culicidae) in urban and rural contexts within Rome Province, Italy. Vector Borne Zoonotic Dis. 2009;10(3):291-4.

46. Vanlandingham D, Higgs S, Huang Yan-Jang S. Aedes albopictus (Diptera: Culicidae) and Mosquito-Borne Viruses in the United States. J Med Entomol. 2016;53(5):1024-8. doi:10.1093/jme/tjw025.

47. Yackulic CB, Reid J, Davis R, Hines JE, Nichols JD, Forsman E. Neighborhood and habitat effects on vital rates: expansion of the barred owl in the Oregon coast ranges. Ecology. 2012;93(8):1953-66. doi:10.1890/11-1709.1.

\section{Submit your next manuscript to BioMed Central and we will help you at every step:}

- We accept pre-submission inquiries

- Our selector tool helps you to find the most relevant journal

- We provide round the clock customer support

- Convenient online submission

- Thorough peer review

- Inclusion in PubMed and all major indexing services

- Maximum visibility for your research

Submit your manuscript at www.biomedcentral.com/submit 